\title{
THE EFFECTIVNESS OF CHRONIC GINGIVITES TREATMENT IN PATIENTS WITH NON-REMOVABLE ORTHODONTIC APPARATUS ACCORDING TO THE RESULTS OF PERIODONTAL TISSUES INDEX ASSESSMENT
}

\author{
Ye. Ya. Kostenko, V. S. Melnyk, L. F. Horzov \\ UZHHOROD NATIONAL UNIVERSITY, UZHHOROD, UKRAINE
}

Background. The risk of chronic gingivitis is increased in patients who undergo orthodontic treatment. It is known that the gingivitis is closely correlated with the level of hygiene.

Objective. The study involved 123 orthodontic patients with chronic catarrhal and hypertrophic gingivitis that developed during the first two months of active orthodontic treatment. We chose VITIS ORTHODONTIC (DENTAID INTERNATIONAL, Spain) that contains active ingredients we need to support healthy state of oral cavity. All studied patients were 12-15 years old.

Methods. For dental examination we used health indices OHI-S according Greene-Vermillion (1964). Gums bleeding was determined according to a modified SBI index by Muhlemann (1971), inflammation of the gingival margin was assessed by PMA index Parma (1960).

Results. Therapeutic treatment consisted of the following: all patients underwent correction of oral hygiene, removal of dental plaque. Vitis ORTHODONTIC was prescribed due to the manufacturer's recommendations: rinse $15 \mathrm{ml}$ for 30 seconds after normal procedures of oral hygiene. Eating or drinking is not recommended during the next 30 minutes after using this product. The results proved a high anti-inflammatory efficacy of the treatment schemes.

Conclusions. The complex therapy of early manifestations of inflammation in periodontal had a positive effect on the subjective feelings of patients and health performance rate, gum inflammation and bleeding.

KEY WORDS: orthodontic patients; chronic gingivitis; dental examination; oral hygiene.

\section{Introduction}

Dentition abnormalities impair the hygienic condition of the mouth, cariogenic effect exacerbate the situation and increase the risk factors of periodontal diseases. Several researchers indicate high percentage of periodontal abnormalities with dentoalveolar lesions $[4,6,9,10]$. Thus, the prevalence of periodontal diseases in patients requiring orthodontic treatment was $81.4 \%$. Due to the Anne-Marie Bollen symptoms of periodontal they were determined in $89.3 \%$ patients. Periodontal tissues were affected in all kinds of bite anomalies [1].

Thus gingivitis is in deep (46.3\%) and an open (43.7\%) occlusion, mesial (37.0\%) and oblique $(33.3 \%)$ bite [2].

The clinical picture may correspond to varying degrees of severity of periodontal tissues diseases. Some authors $[3,5]$ diagnosed

Corresponding author: Lyudmyla Horzov, Children Dentistry Department, Uzhhorod National University, 16a Universytetska Street, Uzhhorod, Ukraine, 88000

Phone number: +380507626129

E-mail: lyudmila_buley@list.ru chronic periodontitis in $70 \%$, gingivitis - in 30\%, including chronic catarrhal gingivitis - in 15\%, medium gravity of hypertrophic gingivitis - in $15 \%$ of people.

The aim of the study was to improve the efficiency of treatment of catarrhal and hypertrophic gingivitis in patients with fixed orthodontic apparatus in mouth when using rinse aid.

\section{Methods}

The examination and treatment of 123 orthodontic patients in permanent dentition period with symptoms of chronic catarrhal and hypertrophic gingivitis that were determined two months after the beginning of active orthodontic treatment by the technique of permanent braces version 'the direct arc'. The patients' age was $12-15$ years: 59 (47.9\%) boys, $64(52.1 \%)$ girls. All patients were almost healthy.

Two weeks before the beginning of orthodontic treatment, all patients were subjected to professional teeth cleaning procedure to remove all deposits and external staining, and 
then tooth surface was polished. Before the beginning of the study instructions on oral hygiene were provided for the patients and a standard method of teeth cleaning was recommended.

In a study patients health indices OHI-S according to Greene-Vermillion (1964) were used. Gums bleeding was determined by a modified SBI index by Muhlemann (1971), inflammation of the gingival margin was assessed by PMA index Parma (1960) [7].

The methods of therapeutic treatment consisted of the following: all patients underwent correction of oral hygiene, removal of dental plaque. Vitis ORTHODONTIC was prescribed due to the manufacturer's recommendations: rinse $15 \mathrm{ml}$ for 30 seconds after normal procedures of oral hygiene. Eating or drinking is not recommended during the next 30 minutes after using this product.

In two weeks of using Vitis ORTHODONTIC twice daily, patients were examined again. When assessing the results of the drug, the views of patients about the taste of the drug, convenience of application was surveyed, we also evaluated the dynamics of the major indexes.

Statistical data processing was carried out by probability Student t-test [8].

\section{Results}

Initial data of periodontal state in the patients before orthodontic phase of treatment are presented in Table 1. Periodontium was clinically healthy. Hygiene was satisfactory.

Within two months from the beginning of orthodontic treatment, due to the lack of oral hygiene, the availability of items for retention of dental plaque around the brackets, most patients complained of bleeding gums when brushing their teeth, swelling, halitosis.

During objective examination, in 70 patients swelling, cyanosis gum, thickening in the area of gingival papilla were evidenced that pointed to a mild severity of catarrhal gingivitis. Mechanical irritation was accompanied by bleeding. In the teeth there was the increased content of soft plaque (patients avoided brushing due to pain and bleeding gums).

According to the results of objective examination, in 53 children hypertrophic gingivitis was determined that proved the visual assessment of size of crown and vertical sensing. All patients had mild severity of hypertrophic gingivitis that was manifested by proliferation of gum to $1 / 3$ crown. To the touch gingival papillae dense produced false periodontal pockets, bleeding was absent, which evidences of the fibrous form of hypertrophic gingivitis.

For gingivitis diagnosis we used classification of periodontal tissues diseases by MF Danilevsky (1994).

Evaluation of patients' performance in two months after the beginning of orthodontic treatment is presented in Table 2 .

All the indicators increased in both groups of patients, oral hygiene index increased to 2.36 points, proving the deterioration in 3 times. The average plaque index increased to 2.6 points that verified unsatisfactory oral hygiene, gingivitis index increased on average by $52 \%$, bleeding index - by $45 \%$.

After the application of hygiene measures and the Vitis ORTHODONTIC solution the patients in 2-3-day reported about reduction of bleeding, gums swelling, disappearance of discomfort events. On examination, the de-

Table 1. Initial data evaluation of the studied patients $(M \pm m)$

\begin{tabular}{|c|c|c|}
\hline Indicators & $\begin{array}{c}\text { Girls } \\
(n=64)\end{array}$ & $\begin{array}{c}\text { Boys } \\
(n=59)\end{array}$ \\
\hline OHI-S (points) & $1.00 \pm 0.04 ; p<0.01$ & $1.10 \pm 0.03 ; p<0.01$ \\
\hline PMA (\%) & $18.3 \pm 2.8 ; p<0.05$ & $9.9 \pm 1.2 ; p<0.05$ \\
\hline SBI $(\%)$ & $7.70 \pm 0.02 ; p<0.01$ & $8.50 \pm 0.12 ; p<0.01$ \\
\hline
\end{tabular}

Note. Statistical significance of differences between the relevant groups of girls and boys.

Table 2. Evaluation of the patients examination in two months after the beginning of orthodontic treatment $(\mathrm{M} \pm \mathrm{m})$

\begin{tabular}{|c|c|c|}
\hline Indicators & $\begin{array}{c}\text { Chronic catarrhal gingivitis, } \\
\text { mild severity } \\
(n=70)\end{array}$ & $\begin{array}{c}\text { Chronic hypertrophic gingivitis, } \\
\text { mild severity, fibrous form } \\
(n=53)\end{array}$ \\
\hline OHI-S (points) & $2.21 \pm 0.04 ; p_{1}<0.05$ & $2.51 \pm 0.03 ; p_{1}<0.05$ \\
\hline PMA (\%) & $69.35 \pm 4.30 ; p_{1}<0.05$ & $71.67 \pm 3.70 ; p_{1}<0.05$ \\
\hline SBI (\%) & $47.17 \pm 0.14 ; p_{1}<0.05$ & $57.16 \pm 0.10 ; p_{1}<0.01$ \\
\hline
\end{tabular}

Note. $\mathrm{p}_{1}$ - difference reliability index compared with the initial data. 
crease of gingival hyperemia was determined. In 8-10 days of the treatment, hyperemia, gingival swelling disappeared, gingival papillae compacted and acquired normal form. By the $14^{\text {th }}$ day of rinses the state of oral health improved significantly. Also with a significant improvement of subjective sensations the pa- tients noted positive dynamics of the indices, and there was no oral mucosa irritant. None of them had any allergic reaction or any adverse side effects. All patients had good rinse results. Teeth sensitivity was not changed. Teeth were not stained. The results are presented in Table 3.

Table 3. Evaluation of patients examination in two weeks after the treatment $(M \pm m)$

\begin{tabular}{|c|c|c|}
\hline Indicators & $\begin{array}{c}\text { Chronic catarrhal gingivitis } \\
(\mathrm{n}=70)\end{array}$ & $\begin{array}{c}\text { Chronic hypertrophic gingivitis } \\
(\mathrm{n}=53)\end{array}$ \\
\hline OHI-S (points) & $0.80 \pm 0.04 ; \mathrm{p} 2<0.05$ & $0.90 \pm 0.03 ; \mathrm{p} 2<0.01$ \\
\hline PMA (\%) & $16.3 \pm 2.8 ; \mathrm{p} 2<0.05$ & $19.9 \pm 1.7 ; \mathrm{p} 2<0.05$ \\
\hline SBI $(\%)$ & $8.20 \pm 0.12 ; \mathrm{p} 2<0.05$ & $10.50 \pm 0.04 ; \mathrm{p} 2<0.05$ \\
\hline
\end{tabular}

Note. $p_{2}$ - differences reliability index compared with the initial data in two months after the beginning of orthodontic treatment.

\section{Discussion}

The results of epidemiological studies of dental status of children in some areas of Ukraine prove great variability and frequency of teeth anomalies, which varies in different regions from $30.8 \%$ to $85.4 \%$ and tends to increase. The treatment of anomalies of jaw apparatus teeth using fixed orthodontic structures is a priority in contemporary orthodontics, because it is highly effective and provides reliable retention of the obtained results. However, in the literature it is mentioned that periodontal tissue react to the treatment with braces, whereby the share of gingivitis according to Petrushanko TA (2013) is 33.3\% [3,4,5]. Among the causes of inflammatory diseases of periodontal tissues in children this categories are defined: worsening of hygienic condition of oral cavity, microbial factors, hormonal changes and the effect of orthodontic forces. However, the

\section{References}

1. Anne-Marie Bollen. Effects of Malocclusions and Orthodontics on Periodontal Health: Evidence from a Systematic Review. Journal of Dental Education. 2008;8:912-918.

2. Benkovskyy W. Clinical assessment of oral hygiene in patients who use orthodontic equipment. 2011.

3. Kudratova DM. Effect of orthodontic treatment on the condition of periodontal tissue. Journal of Dentistry. 2008;1:66.

4. Da Silva Pde L. Gingivitis, psychological factors and quality of life in children. Oral Health Prev Dent. 2015;3:227-235.

5. Lara-Carrillo E. Effect of orthodontic treatment on saliva, plaque and the levels of Streptococcus mutans and Lactobacillus. Med Oral Patol Oral Cir Bucal. 2010;15(6):924-929. development of complex therapeutic measures for chronic catarrhal and hypertrophic gingivitis in children with non-removable orthodontic apparatus has its own significance and importance that should be considered.

Thus, a comparative analysis of clinical trials revealed that the developed complex of therapeutic measures for patients with braces help to improve its clinical course that made it possible to achieve stable remission.

\section{Conclusions}

Inclusion in the complex therapy of early manifestations of inflammation in periodontium had a positive effect on the subjective feelings of patients and health performance rate, gum inflammation and bleeding. It is necessary to emphasize that the use of Vitis ORTHODONTIC must be preceded by correction of oral hygiene and improvement of hygiene practices in patients.

6. Nesterenko OM. Estimated restructuring of jaw bone in adult patients in orthodontic treatment retention period: Poltava; 2008.

7. WHO Bulletin Dental examination, basic methods. Geneva. 1989;3:21.

8. Zajcev VM. Applied Medical Statistics. Foliant. 2006;432.

9. Ostrovska LY. The incidence of periodontal pregnant and mechanisms of development (literature review). Bukovynskiy Medical Journal. 2016;20(77): 215-219.

10. Denga OV. Gingivitis in young people with decreased functional activity of salivary glands. Ukrainian Dental Almanac. 2014;2:26-28.

Received: 2017-01-30 